\title{
Poroid hidradenoma: A Rare Tumor Entity
}

Mohammad Rahmati Roodsari', Hamed Abdolghafoorian², Mohammad Saiedi'

From the Skin Research Center ${ }^{1}$, Shahid Beheshti University of Medical Sciences, Tehran, Iran; Shahid Beheshti University of Medical Sciences ${ }^{2}$, Tehran, Iran.

\section{Abstract:}

Poroid hidradenoma is a rare cutaneous neoplasm, most frequently situated on head and neck. It presents architectural features of hidradenoma, with solid and cystic areas and tumor cells restricted to the dermis, and cytological features of poroid neoplasm such as poroid and cuticular cells. This neoplasm is a new entity which is closely related to proma family. Similar to most of other adnexal tumors, treatment is based on surgical resection in order to prevent a possible recurrence or malignant transformation. To date, very few cases of this entity have been reported in the literature. We present a case of poroid hidradenoma in a 61 -year-old woman with a soft-tissue lesion on her neck.

Key words: Adenoma, Sweat Gland Neoplasms, Acrospiroma, Neck, Neoplasm Recurrence.

\section{Introduction}

Poroid hidradenoma is a benign neoplasm with eccrine differentiation, originally described by Abenoza and Ackerman in 1990 [1]. It is usually a solitary asymptomatic neoplasm that rarely becomes malignant. The onset ranges from 28 to 77 years, with a peak of incidence in the seventh decade. The incidence is approximately equal in male and female patients. The most frequent site of involvement is head and neck. The tumor is typically well circumscribed and wholly intra-dermal, with a diameter ranging from 1 to $2 \mathrm{~cm}$. It appears slightly reddish and tender sensation occurs occasionally. Poroid hidradenoma shows architectural features of hidradenoma and cytologic features of poroid neoplasms in histology. This tumor needs surgical treatment that includes total excision of the lesion in order to prevent its recurrence [2]. Since 1990, less than 20 cases of poroid hidradenoma have been reported in the literature. Here we describe a patient with a poroid hidradenoma of the neck with a rapid growth and a characteristic lobular appearance.

\section{Case Report}

A 61-year-old woman presented to our department with a palpable lesion on the lateral surface of her neck. Family history and past medical history were unremarkable. The lesion had appeared 3 months earlier and rapidly enlarged over a period of two month and easily bleed with minor trauma [Fig.1]. Physical examination revealed a $1 \times 1 \mathrm{~cm}$ non-

\section{Corresponding Author: Dr. Mohammad Saiedi}

Email: rahelehsajadinia@yahoo.com

Received: August 22, 2013 | Accepted: October 30, 2013 | Published Online: December 10, 2013 This is an Open Access article distributed under the terms of the Creative Commons Attribution License (creativecommons.org/licenses/by/3.0)

Conflict of interest: None declared | Source of funding: Nil | DOl: http://dx.doi.org/10.17659/01.2013.0102 
tender, soft red tumor with a vascular appearance and clinically distinct margins. At first the lesion was clinically suspected as being an adnexal tumor or a pyogenic granuloma and then programmed for a radical surgical excision and histological examination. The excised biopsy tumor specimens were fixed in $10 \%$ buffered-formalin and paraffin embedded. 5 sections were provided and stained with haematoxylin eosin, haematoxylin-van Gieson and PAS-haematoxylin for histological examination. Histological examination revealed that the neoplasm was made up of basaloid cuboidal cells with round to oval uniform nuclei arranged in sheets and trabeculae with barely detectable palisading of cells. Some parts showed hyalinization and thickening of the basement membrane. Arrangement of cells at the periphery of vessels and cystic changes with marked plasma cell infiltration were also present. The histological picture described was suggestive of a poroid hidradenoma [Fig.2]. No complication or recurrence was noticed after 3 months of the surgery.

\section{Discussion}

Poroid hidradenoma is an uncommon variant of the eccrine poroma which is a hybrid lesion with features of both hidradenoma and poroma [3]. Typically, poroid hidradenoma presents as a solitary, tender papule or nodule, well circumscribed and wholly intra-dermal, with a diameter ranging from 1 to 2 $\mathrm{cm}$. It appears slightly reddish but the presence of cystic parts may confer a blue color on the lesion caused by the Tyndall phenomenon [4]. The most common sites of involvement are the head and neck regions with a predilection for centro-facial region (about $85 \%$ of cases). Less frequent sites include axilla, trunk and extremities [5].

Poroid hidradenoma is a tumor with solid and cystic components, in which neoplastic poroid cells are all located within the dermis and without connection to the epidermis. This neoplasm presents architectural
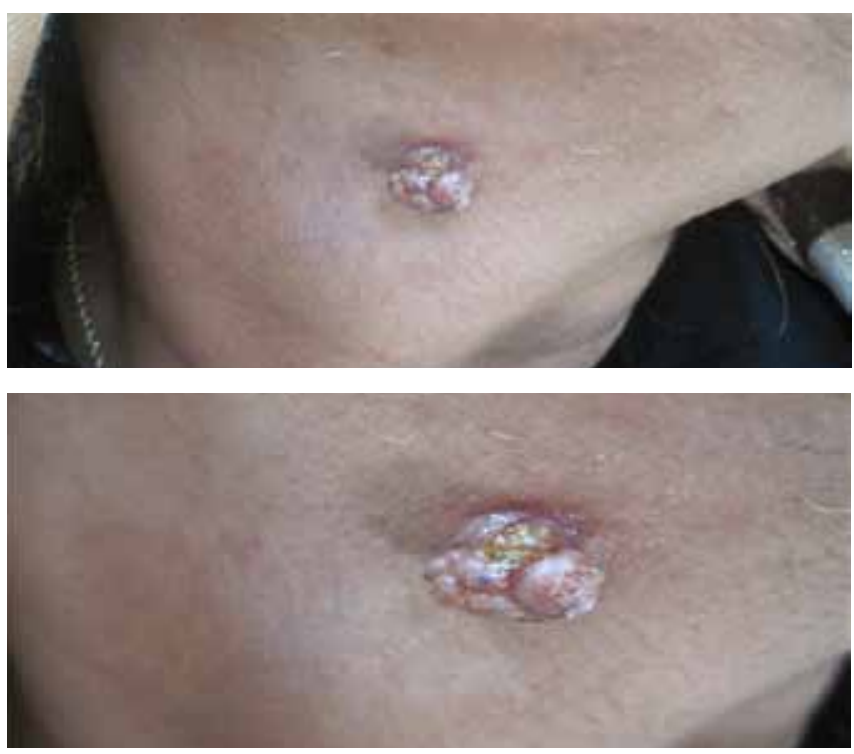

Fig.1: Poroid hidradenoma in a 61 -year-old woman.

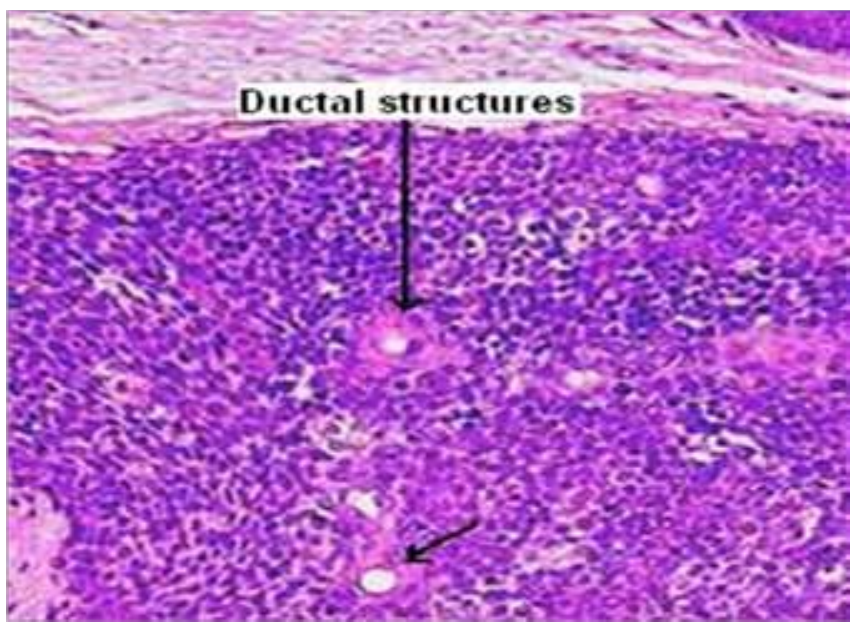

Fig.2: Poroid hidradenoma: histological view; microscopic findings showing a poroid neoplasm with poroid and cuticular cells showing ductal differentiation (HE x 400).

features of hidradenoma, with solid and cystic areas and cytological features of poroid neoplasm such as poroid and cuticular cells [3]. The diagnosis of poroid hidradenoma is based on histological examination of tissue samples. However, there are 
reports of fine needle aspiration cytology (FNAC) value in the diagnosis of cutaneous lesions especially in cases with cyst formation [6].

The main clinical differential diagnoses of poroid hidradenoma are other members of sweat glands neoplasms such as hidroacanthoma simplex, dermal duct tumor, eccrine poroma, a pocrine hidradenomas. Some benign subcutaneous connective tissue neoplasms such as fibroma, fibrolipoma, dermatofibroma, hemangioma, pyogenic granuloma, epidermal inclusion cyst, basal cell epithelioma and malignant eccrine poroma may also be challenging and may cause confusion with poroid hidradenoma [4]. The tumor in our case had a characteristic lobulated feature and its fragility and bleeding tendency made us to consider pyogenic granuluma as one of our differential diagnosis. Poroid hidradenoma is treated by total excision of the lesion in order to prevent its recurrence. The prognosis of poroid hidradenoma is very good, and recurrence has been reported in few cases [6].

\section{Conclusions}

Poroid hidradenoma is the newest addition to the group of poromas. It is a benign tumor and rarely becomes malignant in less than $1 \%$ of cases, but it can be easily misdiagnosed as a malignant neoplasm. Until now little cases of this entity has been reported in the literature, so the key point in the diagnosis is to be in thinking about it and taking an excisional biopsy.

\section{References}

1. Abenoza P, Ackerman AB. Neoplasms with Eccrine Differentiation. Philadelphia, Lea \& Febiger. 1990;1:113-185.

2. Mlika M, Chelly B, Ayadi-Kaddour A, Boudaya S, Kilani T, El Mezni F. Poroid hidradenoma: a case report. Our Dermatol Online. 201 2;3(1):43-45.

3. Requena L, Sanchez M. Poroid hidradenoma: a light microscopic and immunohistochemical study. Cutis. 1992;50:43-46.

4. Delfino S, Toto V, Brunetti B, Di Marino MP, Baldi A, Persichetti P. Poroid hidradenoma: A case report. In vivo. 2007;21:905-908.

5. Yamamoto $O$, Hisaoka $M$, Yasuda $H$, Kasai T, Hashimoto $H$. Cytokeratin expression of apocrine and eccrine poromas with special reference to its expression in cuticular cells. J Cutan Pathol. 2000;27:367-373.

6. Hoshida Y, Hanai J, Matsushita N, Yonekawa $M$, Kobayashi $Y$, Kawakami $H$, et al. Poroid hidradenoma: Report of a case with cytologic findings on fine needle aspiration. Acta Cytol. 1999;43:471-474. 